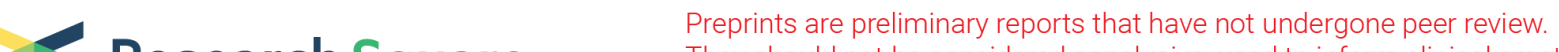 Research Square They should not be considered conclusive, used to inform clinical practice, or referenced by the media as validated information.
}

\section{Mathematical modeling and comparative analysis of the Ebola epidemic reveal effective control measures}

\author{
Junlong Li
}

Southwest Hospital『Chongqing, China

\section{Caiping Song}

Xinqiao Hospital,Chongqing,China

\section{Jingya Yang}

College of Mathematics and Statistics, Chongqing University,Chongqing

\section{Jingmin Zheng}

Southwest Hospital

\section{Lina Zhou}

Southwest Hospital

\section{Zhujun Zhang}

Southwest Hospital

Jie Pu

Southwest Hospital

Yang Luo

Southwest Hospital

Hongyan Zhang

Southwest Hospital

\section{Yungui Wang}

Southwest Hospital

Luosheng Wen ( $\nabla$ wls@cqu.edu.cn )

https://orcid.org/0000-0003-4308-9802

Hao Wu

Southwest Hospital

\section{Research article}

Keywords: Ebola virus disease, Ebola virus, mathematical modeling, comparative analysis, prevention

Posted Date: September 17th, 2019

DOI: https://doi.org/10.21203/rs.2.14493/v1 
License: (c) (i) This work is licensed under a Creative Commons Attribution 4.0 International License. Read Full License 


\section{Abstract}

The Ebola virus disease (EVD) is a highly contagious disease which is caused by the Ebola virus . Various measures were used to prevent and control the spread of EVD. The aim of this study was to find out the most critical measures to prevent and control the spread of EVD. Both mathematical modeling and comparative analysis were used to explore the development process of EVD outbreak in Guinea, Liberia and Sierra Leone. The results of comparative analysis showed quarantined individuals before infection ( $\mathrm{R}$ $2=0.848, \varepsilon=1.012)$, safe burial teams $(\mathrm{R} 2=0.772, \varepsilon=0.385)$, and the Ebola treatment units (ETU) bed ( $R 2=0.690, \varepsilon=0.432$ ) could significantly influence the incidence of EVD which were consistent with the results of mathematical modeling. These findings indicted that a timely and effective quarantine played a significant role in preventing and controlling the spread of EVD, and the findings would help us prevent and control the epidemic outbreak of new infectious disease in the future.

\section{Introduction}

Ebola virus disease (EVD), previously known as Ebola hemorrhagic fever, is caused by infection of the Ebola virus. Beginning in December 2013 in West Africa, precisely in Guinea, the EVD outbreak spread to Sierra Leone and Liberia at the fatality rates of $73.2 \%, 43.0 \%$ and $52.5 \%$, respectively[1].Until now, EVD has been known to be a highly contagious disease with a high mortality rate. Ebola virus is classified as a biosafety level 4 agent by the World Health Organization (WHO) [2, 3] A total of 28,616 Ebola cases and 11,310 deaths have been reported in Guinea, Liberia and Sierra Leone[4].

Mathematical modeling has contributed to the investigation of EVD dynamics in recent years. Chowell proposed a stochastic SEIR model to fit the data of EVD outbreaks from 1995 to 2000 in Congo and Uganda, respectively[5]. They were able to determine the basic reproduction numbers of the EVD spread in the two countries. Althaus applied the SEIR model to study the reproduction numbers of the 2014 EVD spread and predicted the control measure effects through the results obtained[6, 7]. Following these studies, the specific circumstances of the infectious disease spread were taken into account. For example, thanks to improvements in medical treatment, management, and monitoring, mathematical models were improved by adding these components. Adnan Khan established the $S_{L} S_{H} E I H R$ model and obtained the reproduction numbers for Liberia and Sierra Leone[1]. In that model, the susceptible individuals were divided into low-risk $\left(\mathrm{S}_{\mathrm{L}}\right)$ and high-risk $\left(\mathrm{S}_{\mathrm{H}}\right)$ groups according to their geographical differences. According to the data of Congo and Uganda, a SEIHFR model was established in which $\mathrm{H}, \mathrm{F}$, and $\mathrm{R}$ represented the hospitalized status, dead (not buried) patients, and buried patients, respectively[8]. Camacho proposed a $\mathrm{SE}_{\mathrm{pp}} \mathrm{E}_{\mathrm{h}} \mathrm{IHDBR}$ model and estimated that the human-to-human reproduction number was 1.34 in the EVD early phase[9]. Especially, they suggested that Ebola control would be difficult when counting on hospital-based control measures alone.

Recently, some researchers focused on the control measures in the breakout and analyzed the effect of the measures. Pandey et al. highlighted the Ebola spread in funerals through different airspace compartments that represented different groups[10]. They considered that control measures at different 
times had different impacts, which allows for further discussion. Lewnard et al. developed a model to assess the effectiveness of expanding EVD treatment centre, increasing case ascertainment, and allocating protective kits for controlling the outbreak in Montserrado[11]. The number of treatment beds and estimated how many cases were averted as a result of the introduction of additional treatment beds in each area[12]. Otherwise, an SEIR type model which characterizes the impact of contact tracing on the effective reproduction number of Ebola was represented[13, 14].

To estimate the control measures adopted by the three countries and analyze which types of efforts they should make, we propose a $S E_{f} E_{c} l_{f} l_{C} l_{C C} R$ model, in which the exposed and infectious individuals are classified into several compartments according to their controlled situations to assess the intervention strategies. We used the weekly data from the WHO and some parameters to fit the efficiencies of the preinfected and post-infected control measures. Our results showed that pre-infected and post-infected control measures in Liberia were the best among the three countries of West Africa. Further analysis showed that more effort should be made in Guinea because the relative change rate is the largest for the two parameters.

\section{Results}

\section{Numerical results and analysis}

Using the model and data mentioned above, we obtained the numerical results for the three countries' predicted infected numbers (Figure-1).. The blue curve is a SEIR model run fitting early phase weekly data. It is clear that the three maximum numbers of new daily cases were up to about $290,1,100$, and 1,300 in Guinea, Liberia, and Sierra Leone, respectively, which were far more than the confirmed Ebola incidence according to the WHO (171, 364 and 565, in the same order). This result implies that the Ebola prevention and control measures are urgent and important. The black curve is a data fitting result using the proposed model.

We also obtained the basic reproduction number of the system (8). We obtain the basic reproduction number $R=(1-a)((1-c) \beta / \gamma$ to control measures according toDriessche PV[15]. The estimated parameters in the model are shown in Table 2. A higher value of a means that a larger proportion of highrisk individuals in the country were quarantined thanks to the pre-infected measures, such as common knowledge of Ebola, early diagnosis, and contact tracing. The results showed that Liberia's pre-infected measure was the most efficient, followed by Sierra Leone, and then Guinea. Meanwhile, a larger value for $c$ means that a larger proportion of infectious individuals in the country could be identified and isolated earlier by post-infected measures. Moreover, it is clear that the post-infected measures in Liberia work better than those in Guinea and Sierra Leone (the parameter $c$ of Liberia is twice that of the latter two). Therefore, the results showed that Liberia achieved the best outcome while Guinea had the worst in terms of pre-infected and post-infected control measures. 
To show the efficiency of the two control parameters, we determined the numerical results for the relation between the accumulated infected number in the model and the two control parameters. In Figure-2, the predicted infected number is a decreasing function of the two parameters. For the three countries, the accumulated number will sharply decrease in the field with a small $a$ and $c$. It is shown that the parameters of Guinea are in the sharply decreasing field while those of Liberia are in the gently decreasing field. This means that Liberia has conducted a more efficient control strategy and Guinea's control strategy needs improvement.

We further studied the sensitivity of parameters $a$ and $c$. We fixed one parameter at the fitting values shown in Table 2 and varied the other parameter. As shown in Table 3, we can find Guinea had the largest relative change rates for $a$ and $c$, which means that small improvement in the control strategy will sharply decrease the accumulated infected number.

The influences of different measures on the increase rates of new EVD cases in the three countries in West Africa

Based on the information on EVD spread control measures in the situation reports provided by the WHO, we obtained the following data: the number of Ebolatreatment units (ETU) beds, the number of suspected cases and probable cases, which we defined as the quarantine number before the disease, and the number of safe burial teams. Then we accumulated the information after quantifying the implementation of the three measures. First, we conducted a correlation analysis among the three measures above and the weekly EVD increase rates in the three countries, respectively (Figure-4, Figure-5 and Figure-6). The new case increase rates were all negatively correlated with the three measures, with a statistically significant difference. The number of weekly ETU beds, the quarantine number before infection, and the number of safe burial teams were modeled separately with the increase rates of EVD new cases, the results of which achieved a goodness of fit. The $R^{2}$ were $0.690(P<0.001), 0.848(P<0.001)$, and 0.772 $(P<0.001)$, respectively. Moreover, to know which of the three measures had the greatest impact on the increase rates of new cases, we conducted a multivariate linear regression analysis to identify the most relevant factors against the overall backdrop. The results are shown in Table 4. The absolute values of the standardized parameters of quarantine measures before infection are first $(\varepsilon=1.012, P<0.01)$, that of increasing the ETU bed number follows $(\varepsilon=0.432$, P $<0.05)$, and that of increasing the safe burial teams was the smallest $(\varepsilon=0.385, \mathrm{P}<0.05)$.

All of the three measures had a favorable linear relationship with the increase rates of new cases. The results of multivariate linear regression analysis indicated that expanding the quarantine before disease was the most effective control measure. The following figures demonstrate the results above. Apparent differences were observed in the quarantine number before disease among the three countries (Figure -7 , Figure- 8 and Figure-9).. Within the statistical data we obtained, the ranking of the maximum proportion of the quarantine number before disease of the total quarantine number was as follows: Liberia $(79.31 \%)$ $>$ Sierra Leone (31.35\%) > Guinea (25.62\%), and the differences among the three countries were all statistically significant $(P<0.05)$. 


\section{Discussion}

The outbreak of EVD began in December 30, 2013 in Guinea, and it was considered as the largest, the most serious, and the most complex international crisis by the WHO[16]. Then, it spread into three countries (Liberia, Guinea, and Sierra Leone) in West Africa within a very short time, although they were not first occurrence locations. The climate environment, religion, customs, poverty, population density and so on, are all potential factors causing the outbreak of Ebola virus in West Africa. Contact was the main route of transmission for EVD.

In this study, we mainly evaluated the effects of Ebola intervention strategies. By comparing the infected number of patients before and after the interventions through the model, it is evident that timely and accurate intervention strategies, such as quarantine ability, isolation, and hospitalization, can effectively control the outbreak and transmission of EVD in the three countries in West Africa. However, the most important intervention is quarantine, which has an obvious influence on infection rates. Our model is designed to capture the key characteristics of Ebola spread. Its computation is an innovative result for Ebola disease models, which has not been presented before and will be useful for investigations of effective epidemic control measures in the future as well. The three countries' prevention and control measures in West Africa were estimated and compared. Following our paper, further work can continue with following aspects: the development and impact of an Ebola vaccine on prevention and control measures; the effects of population behaviors, such as commerce, tourism and others; detailed numbers of hospitals, ETUs and isolation in the community; more accurate analysis of the effects of specific prevention and control measures; the availability of beds in ETUs estimated from the observed data; the limitations in the amount and timely payment numbers of hospital patients who could be accommodated; and the allocation of Ebola patient beds and so on.

In addition, to determine the effects of $a$ and $c$ obtained in the model in practical epidemic situation control, we classified the control measures in the situation reports $₫$ the measures related to $a$ included the ETU bed number, Community Care Centre (CCC), common knowledge of Ebola, laboratory capacity, effective quarantine aimed to probable and suspected patients, and contact tracing. All of these were targeted at probable and suspected individuals without a definite diagnosis. The measures related to $c$ included laboratory capacity, effective quarantine aimed at patients with a definite diagnosis, and the number of safe burial teams for dead patients, the objectives of which were the infected individuals. However, incomplete data on the control measures during the epidemic expansion imposed restrictions on the integrated and comprehensive analysis. Consequently, although we can only conduct quantitative analysis on the ETU bed numbers, the number of quarantined individuals before infection, and the numbers of safe burial teams, the three measures act as the core measures in epidemic situation control.

The effectiveness ranking of the three measures we obtained from the multivariate regression analysis was: quarantine before disease > the number of ETU beds > safe burial, which were consistent with the results of our model. The epidemic situation control in Liberia is the best among the three countries in West Africa. A person who has (or had) sudden onset of a high fever and at least three of the following 
symptoms: headache, vomiting, anorexia/loss of appetite, and breathing difficulties, was classified as a suspected case. Any suspected case evaluated by a clinician or any person who died from 'suspected' EVD and had an epidemiological link to a confirmed case but was not tested and did not have laboratory confirmation of the disease, was classified as a probable patient. A definite diagnosis of this kind of patient requires a great number of medical care personnel with professional knowledge. In addition, epidemic situation knowledge delivered by international or local health organizations is needed to inform people that someone with the above-mentioned symptoms belongs to the high-risk population and needs go to the local ETU or CCC on his own initiative to receive diagnosis and treatment as well as transformation to the ETU after a definite diagnosis[17, 18]. As a joint effort by the WHO and the Ministries of Health of the three countries in West Africa, a CCC is a new strategic pattern in response to EVD spread. It is aimed to perform an effective quarantine on the suspected, probable and confirmed cases when the ETU is overloaded. When people are diagnosed, as in the cases above, they will be quarantined in the CCC or ETU with sufficient bearing capacity and looked after by professional medical care personnel[17, 19]. Reports have confirmed that a powerful intervention measure to prevent the Ebola virus from spreading in Liberia is to quarantine the confirmed cases and probable and suspected patients into the ETU. When the ETU is overloaded or not available, quarantining the patient into the communitybased infrastructures, such as the CCC, could reduce the risk of EVD spread[20,21]. An obvious advantage of such a measure is enabling the government to rapidly quarantine all the possible patients who might become infectious sources and monitor them for contact tracing. Thus, the risk of infecting others could be minimized when the patient is attacked by EVD[22]. It cannot be neglected that the CCC still carries the potential risk of spreading EVD. The virus could spread between patients and medical care personnel or among other personnel in the community when someone with the virus has not been diagnosed in time by the laboratory or the medical care. Personnel have exposed themselves to a high risk environment because of incautious operations[23]. However, the CCC plays a crucial role in preventing the spread of EVD[24].

A person who has (or had) sudden onset of a high fever and at least three of the following symptoms: headache, vomiting, anorexia/loss of appetite, breathing difficulties, will be classified as a suspected case. Any suspected case evaluated by a clinician or any person who died from 'suspected' EVD and had an epidemiological link to a confirmed case but was not tested and did not have laboratory confirmation of the disease, will be classified as a probable patient. This reminded us that during the disease spreading period, especially those infectious diseases transmitted via contact, the priority of controlling the spread is to recognize and distinguish the probable or suspected patients as soon as possible, so as to take effective quarantine measures before disease spread. It is notable that without favorable communication between medical care personnel and patients, as well as the Ebola epidemic knowledge popularization, the arbitrary activity restrictions on probable or suspected patients posed by government unilaterally will lead to panic or even conflicts.

Admittedly, besides the three measures discussed in this paper, there are many measures that could have effects on the epidemic situation. For example, the laboratory capacity will influence the detection rate and speed of patients' blood sample testing and in this way it will influence the reaction time of taking 
effective measures such as quarantine and treatments on real EVD patients as well as self-protection of medical care workers; the number of professional personnel involved in contact tracing could influence the probability of second transmission by infected people, thus affecting transmission control. However, because of data deficiency, quantitative analysis in these regards will not be included.

\section{Materials And Methods}

\section{The $S E_{f} E_{c} l_{f} l_{c} l_{f} R$ model}

For the early phase of EVD outbreak when few efficient control measures are taken, we only used the classical SEIR model as the early dynamic model. For the middle and late phases of the epidemic, a new model was needed to capture the key characteristics of the control measures.

The population transmission diagram of the model is depicted in Figure-10. Considering the quarantine and isolation measures, the compartments $E_{f} I_{c}$ and $l_{f c}$ were added to the classical SEIR model. The subscript $c$ represents the control state (quarantined or isolated), and $g$ stands for the control-free state. For example, the compartment $E_{c}$ represents the quarantined latent individuals. An individual with state $E_{c}$ will naturally become an individual with an isolated infectious state after several days, that is, $I_{c}$. The subscript $f c$ represents the isolated status after a control-free status. $I_{f C}$ stands for those previously control-free individuals who were infected and then were sent to a hospital for isolation and treatment after further Ebola testing and diagnosis due to their Ebola symptoms.

In Figure-10, the parameter a stands for the proportion of susceptible individuals who become the controlled latent individuals $\left(E_{c)}\right.$. The parameter describes the ability of a country and the government to identify and quarantine high-risk individuals. A larger value for a means that a government can more efficiently identify a high-risk individual before the person is infectious. The parameter $c$ represents the proportion of individuals who turn from $E_{f}$ to $I_{f c}$. The parameter describes the ability of a country and the government to control the epidemic for the infected individuals. Also, a larger value of $c$ means that people can isolate the infective individuals earlier. The system's differential equations are shown as follows:

\section{Data sources}

Data fitting is done using the weekly confirmed incidence data reported by the WHO in Guinea, Liberia and Sierra Leone[13]. The value of $I(t)$ is the sum of the compartments $I_{f,} I_{f C}$ and $I_{C}$ in the model.

\section{Parameter values}

The parameters $\sigma$ and $y$ in the model can be obtained from published papers (or data), as described in Table 1. 


\section{Estimation scheme}

To obtain the infection rate $\beta$ in the classical SEIR model, we used the single-stepalgorithm-Runge-Kutta method of order 4 with good precision and speed. The method's formulas are shown as follows:

The SEIR model and preliminary data were used to determine the infection rate $\beta$ in the three countries. To solve the optimal parameter value, the command fmincon was used in Matlab. We ran a numerical simulation of $\beta$ with the constraint condition $0<\beta<1$ in the least-squares (OLS) sense. The objective function isin which $d$ is the weekly Ebola incidence data in the early phase by WHO and $m$ is the weekly

corresponding data obtained. The confirmed cases of the three countries before $40^{\text {th }}$ week, $25^{\text {th }}$ week and $24^{\text {th }}$ week are used.

In the middle and late phases of the outbreak, that is, the period after $40^{\text {th }}$ week, $25^{\text {th }}$ week and $24^{\text {th }}$ week for the three countries, we used the $S E_{f} E_{c} l_{f} l_{c} l_{f c} R$ model and the data of the three countries to fit the values of parameter $a$ and $c$. We fixed the parameters mentioned above and then solved the parameters $a$ and $c$ by fmincon in the objective function with constraints $0 \leq a \leq 1$ and $0 \leq c \leq 1$.

\section{The comparison analysis of the control measures}

To determine which kind of measure is the most effective for preventing EVD spread for pre-infected and post-infected individuals, we conducted a correlation analyses between the growth rates of the confirmed cases and all the measures used in West Africa. We used the published data from situation reports from the WHO from August 2014 to May 2015 over the course of the outbreak. This time period included all the phases of the EVD epidemic, from the rapid spread of EVD; that is, the height of the outbreak, to the time when the epidemic situation has been gradually controlled. Throughout this period, the control measures become comprehensive and standardized step by step; however, it is notable that there are differences between the three countries in the effects of the epidemic situation control. We evaluated the correlation between the measures adopted in different phases and increases in the rates of EVD. We estimated it from the $34^{\text {th }}$ week of the epidemic outbreak when the WHO first issued an Ebola epidemic situation report (August 29,2014 ) to the $70^{\text {th }}$ week when the epidemic situation had been apparently curbed (May $13,2015)$. The reason why we chose this time period for the study is that it includes the gradual increaseto-decrease process of intervention measures that are described in detail, despite some data deficiency. On the basis of having confirmed the favorable linear relationship between different measures and the increase rates of new cases, respectively, we used a multivariate linear regression function to compare the effect of intensities of different measures on the increase rates of new cases.

\section{Declarations}

SEIR model: susceptible-exposed-infected-recovered model; 
$S_{L} S_{H} E I H R$ model: low-risk susceptible individuals, high-risk susceptible individuals, exposed individuals, infected individuals, hospitalized individuals, and recovered individuals R;

SEppEhIHDBR model: susceptible to infection (S); entering an incubation period (E); symptomatic and infectious in the community (I); entering a recovered state (R); remain infectious and go into hospital $(H)$; or die and remain infectious (D) until buried (B).

\section{Acknowledgements}

This work was supported by grants from the National Natural Science Foundation of China $(71540020$, 71373280).

\section{AUTHOR CONTRIBUTIONS}

Junlong Li and Caiping Songdid statistical analysis and wrote the original manuscript, they contributed equally to this work and listed as the first authors. Luosheng Wen and Hao Wu conceived the study and obtained financial support. Jingya Yang established the mathematical model. Jingmin Zheng, Lina Zhou, Zhujun Zhang, Jie Pu, Yang Luo, Hongyan Zhang and Yungui Wang did the literature search and data collection. All authors have reviewed and approved the final manuscript.

\section{Additional Information}

\section{Competing interests}

The author(s) declare no competing interests.

Data Availability

The datasets generated or analysed on the current study are available on reasonable request from the corresponding author.

\section{References}

1.Khan A, Naveed M, Dur-E-Ahmad M, Imran M: Estimating the basic reproductive ratio for the Ebola outbreak in Liberia and Sierra Leone. INFECT DIS POVERTY2015, 4:13.

2.Ki M: What do we really fear? The epidemiological characteristics of Ebola and our preparedness. Epidemiol Health 2014, 36:e2014014.

3.Na W, Park N, Yeom M, Song D: Ebola outbreak in Western Africa 2014: what is going on with Ebola virus? Clin Exp Vaccine Res 2015, 4(1):17-22. 
4.Cleaton JM, Viboud C, Simonsen L, Hurtado AM, Chowell G: Characterizing Ebola Transmission Patterns Based on Internet News Reports. CLIN INFECT DIS 2016, 62(1):24-31.

5.Chowell G, Hengartner NW, Castillo-Chavez C, Fenimore PW, Hyman JM: The basic reproductive number of Ebola and the effects of public health measures: the cases of Congo and Uganda. J THEOR BIOL 2004, 229(1):119-126.

6.Althaus CL: Estimating the Reproduction Number of Ebola Virus (EBOV) During the 2014 Outbreak in West Africa. PLoS Curr 2014, 6.

7.Althaus CL, Low N, Musa EO, Shuaib F, Gsteiger S: Ebola virus disease outbreak in Nigeria:

Transmission dynamics and rapid control. EPIDEMICS-NETH 2015, 11:80-84.

8.Legrand J, Grais RF, Boelle PY, Valleron AJ, Flahault A: Understanding the dynamics of Ebola epidemics. EPIDEMIOL INFECT 2007, 135(4):610-621.

9.Camacho A, Kucharski AJ, Funk S, Breman J, Piot P, Edmunds WJ: Potential for large outbreaks of Ebola virus disease. EPIDEMICS-NETH2014, 9:70-78.

10.Pandey A, Atkins KE, Medlock J, Wenzel N, Townsend JP, Childs JE, Nyenswah TG, Ndeffo-Mbah ML, Galvani AP: Strategies for containing Ebola in West Africa. SCIENCE 2014, 346(6212):991-995.

11.Lewnard JA, Ndeffo MM, Alfaro-Murillo JA, Altice FL, Bawo L, Nyenswah TG, Galvani AP: Dynamics and control of Ebola virus transmission in Montserrado, Liberia: a mathematical modelling analysis. LANCET INFECT DIS 2014, 14(12):1189-1195.

12.Kucharski AJ, Camacho A, Flasche S, Glover RE, Edmunds WJ, Funk S: Measuring the impact of Ebola control measures in Sierra Leone. Proc Natl Acad Sci U S A 2015, 112(46):14366-14371.

13.Browne C, Gulbudak H, Webb G: Modeling contact tracing in outbreaks with application to Ebola. J THEOR BIOL 2015, 384.33-49.

14.Browne C, Gulbudak H, Webb G: Modeling contact tracing in outbreaks with application to Ebola. J THEOR BIOL 2015, 384:33-49.

15.van den Driessche P, Watmough J: Reproduction numbers and sub-threshold endemic equilibria for compartmental models of disease transmission. MATH BIOSCI 2002, 180.29-48.

16.Gostin LO, Friedman EA: A retrospective and prospective analysis of the west African Ebola virus disease epidemic: robust national health systems at the foundation and an empowered WHO at the apex. LANCET 2015, 385(9980):1902-1909.

17.Logan G, Vora NM, Nyensuah TG, Gasasira A, Mott J, Walke H, Mahoney F, Luce R, Flannery B: Establishment of a community care center for isolation and management of Ebola patients - Bomi 
County, Liberia, October 2014. MMWR Morb Mortal Wkly Rep 2014, 63(44):1010-1012.

18. Whitty CJ, Farrar J, Ferguson N, Edmunds WJ, Piot P, Leach M, Davies SC: Infectious disease: tough choices to reduce Ebola transmission. NATURE 2014, 515(7526):192-194.

19.Olu O, Cormican M, Kamara KB, Butt W: Community Care Centre (CCC) as adjunct in the management of Ebola Virus Disease (EVD) cases during outbreaks: experience from Sierra Leone. Pan Afr Med J 2015, 22 Supp/ 1:14.

20.Nyenswah T, Fahnbulleh M, Massaquoi M, Nagbe T, Bawo L, Falla JD, Kohar H, Gasasira A, Nabeth P, Yett S et al: Ebola epidemic-Liberia, March-October 2014. MMWR Morb Mortal Wkly Rep 2014, 63(46):1082-1086.

21.Michaels-Strasser S, Rabkin M, Lahuerta M, Harripersaud K, Sutton R, Ahoua LN, Ngalamulume B, Franks J, El-Sadr WM: Innovation to confront Ebola in Sierra Leone: the community-care-centre model. LANCET GLOB HEALTH2015, 3(7):e361-e362.

22.Michaels-Strasser S, Rabkin M, Lahuerta M, Harripersaud K, Sutton R, Ahoua LN, Ngalamulume B, Franks J, El-Sadr WM: Innovation to confront Ebola in Sierra Leone: the community-care-centre model. LANCET GLOB HEALTH2015, 3(7):e361-e362.

23.Fischer WN, Hynes NA, Perl TM: Protecting health care workers from Ebola: personal protective equipment is critical but is not enough. ANN INTERN MED 2014, 161(10):753-754.

24.Kucharski AJ, Camacho A, Checchi F, Waldman R, Grais RF, Cabrol JC, Briand S, Baguelin M, Flasche S, Funk S et al: Evaluation of the benefits and risks of introducing Ebola community care centers, Sierra Leone. EMERG INFECT DIS 2015, 21(3):393-399.

\section{Tables}

Table 1. The parameters used in the models.

\begin{tabular}{lllll}
\hline Country & Parameter & Meaning & Value & References \\
\hline Guinea & $1 / \sigma$ & the incubation period & 10.9 & {$[5]$} \\
& $1 / \gamma$ & the infection period & 6.4 & {$[5]$} \\
Liberia & $1 / \sigma$ & the incubation period & 11.7 & {$[5]$} \\
& $1 / \gamma$ & the infection period & 7.9 & {$[5][13]$} \\
Sierra Leone & $1 / \sigma$ & the incubation period & 10.8 & {$[5]$} \\
& $1 / \gamma$ & the infection period & 6.7 & {$[5][13]$} \\
\hline
\end{tabular}


Table 2. The parameter values in the model.

\begin{tabular}{lllll}
\hline Country & $\beta$ & Parameter & Value & $R$ \\
\hline Guinea & 0.2131 & $a$ & 0.1827 & 0.9930 \\
& & $c$ & 0.1091 & \\
Liberia & 0.2414 & $a$ & 0.5832 & 0.5343 \\
& & $c$ & 0.3278 & \\
Sierra Leone & 0.2062 & $a$ & 0.3696 & 0.7593 \\
& & $c$ & 0.1281 & \\
\hline
\end{tabular}

Table 3. The relative change rate in the three countries.

\begin{tabular}{lrr}
\hline Liberia & -0.1750 & -0.1746 \\
Sierra Leone & -0.1628 & -0.1726 \\
Guinea & -0.5139 & -0.5050 \\
\hline
\end{tabular}

Table 4. Standardized parameters of the three measures.

\begin{tabular}{lll}
\hline Measure & Standardizedparameter $\varepsilon$ & $\mathrm{P}$ value \\
\hline Established ETU bed number & 0.424 & $\mathrm{P}<0.05$ \\
Quarantine before infection & 1.025 & $\mathrm{P}<0.01$ \\
Safe burial team number & 0.364 & $\mathrm{P}<0.05$ \\
\hline
\end{tabular}

Figures 
A

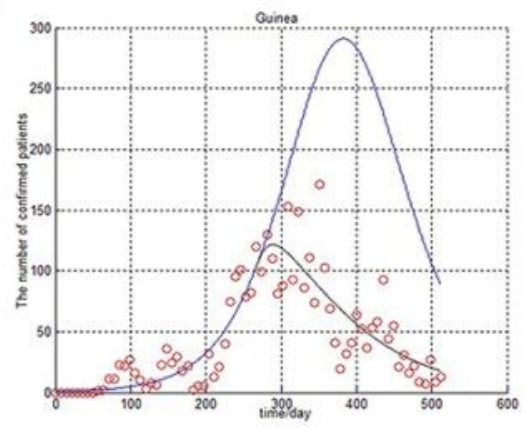

B

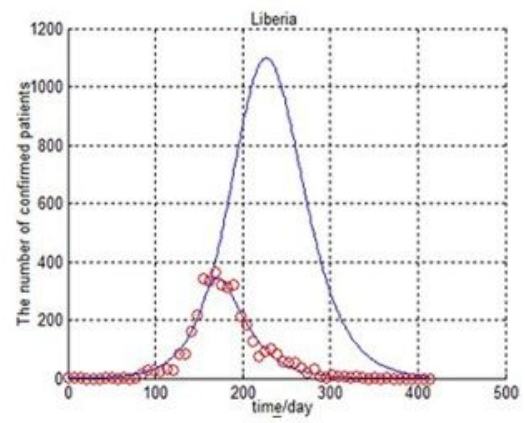

C

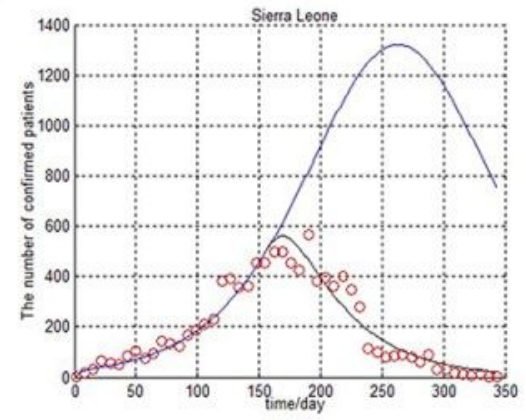

Figure 1

Ebola cases in West Africa with isolation measures in the middle and late phases
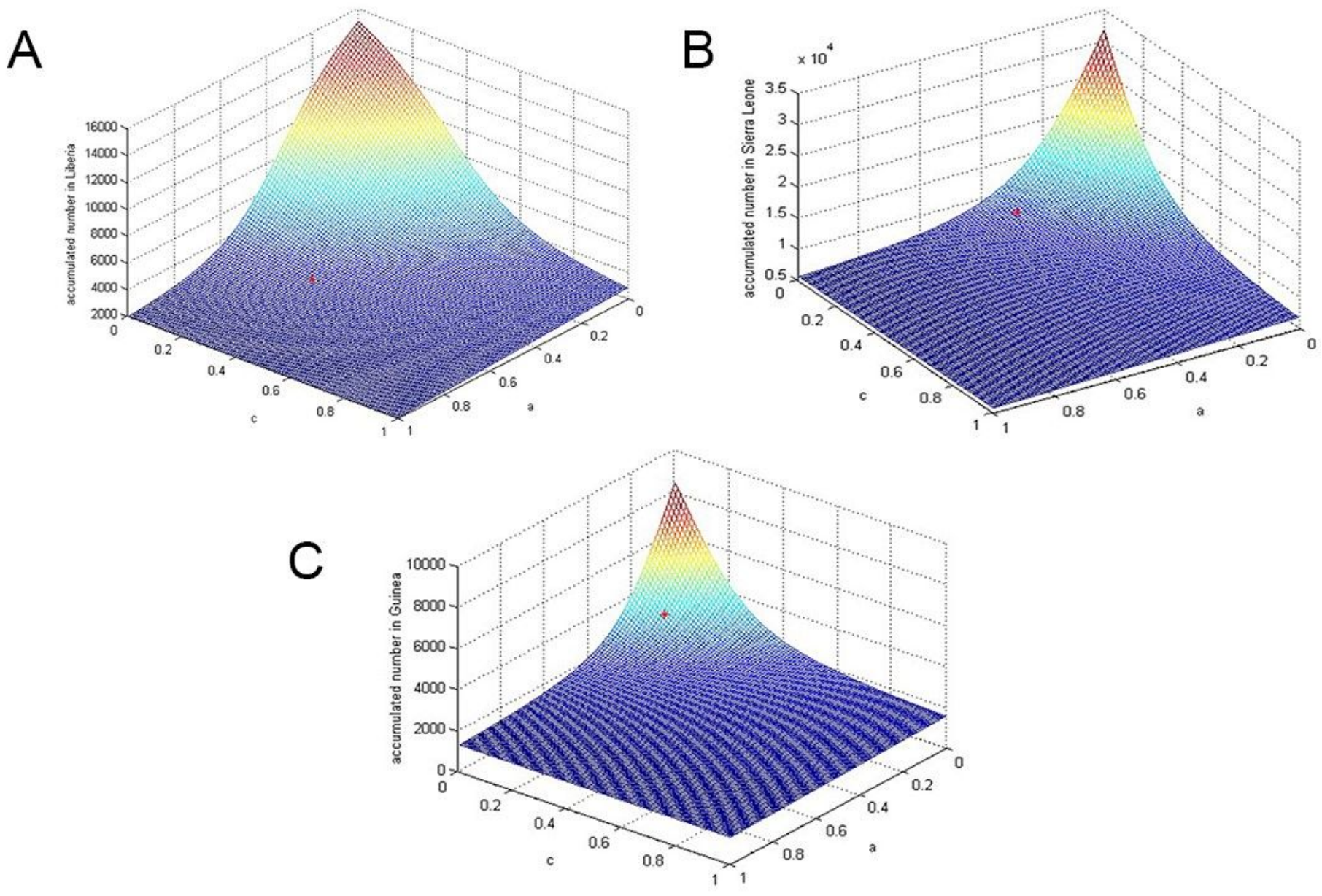

Figure 2

The predicted accumulated infected number in the model 

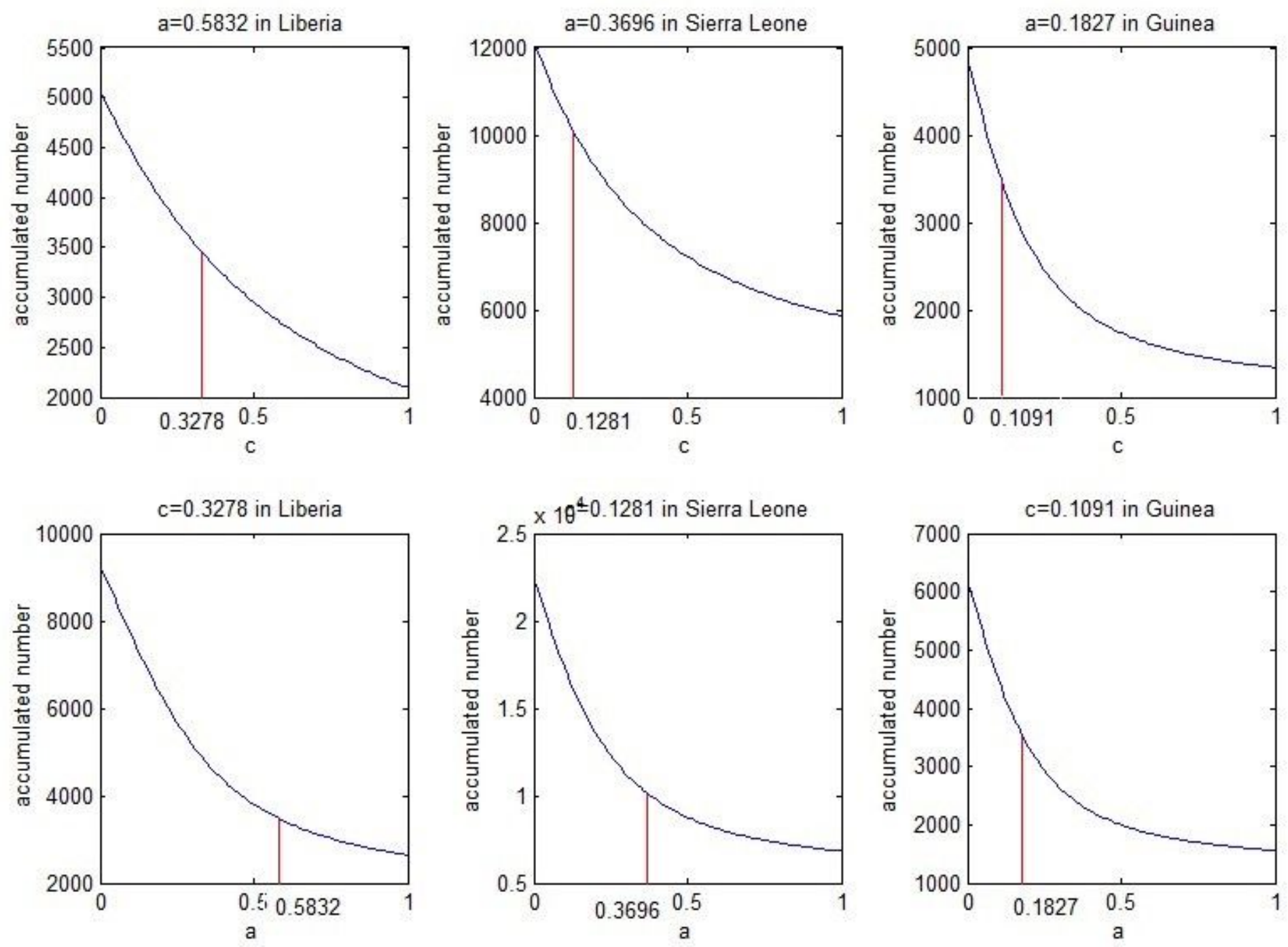

\section{Figure 3}

The sensitivity of parameters a and $\mathrm{c}$ in the three countries 


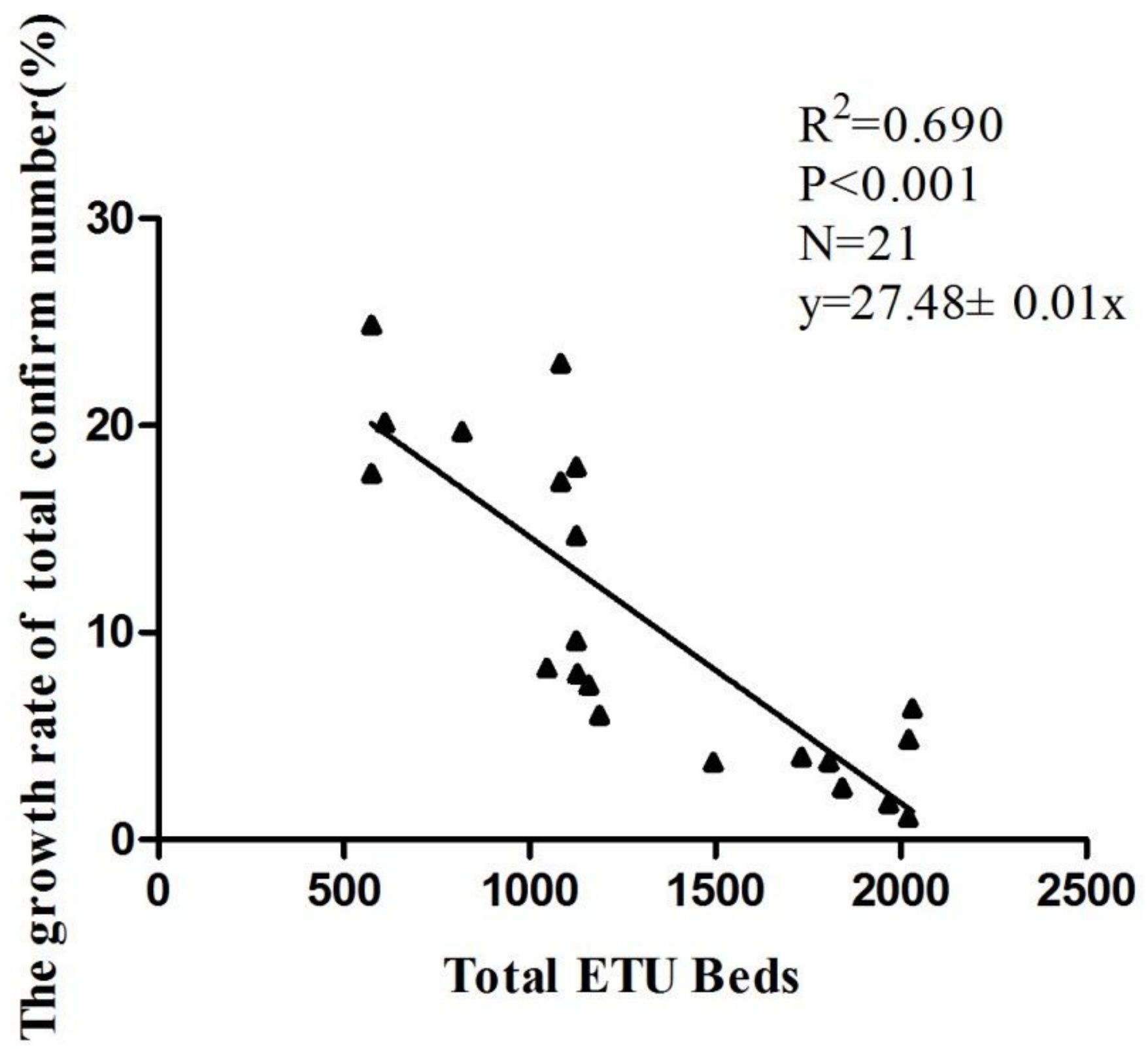

Figure 4

The correlation analysis between established ETU beds and the weekly EVD increase rates in Guinea 


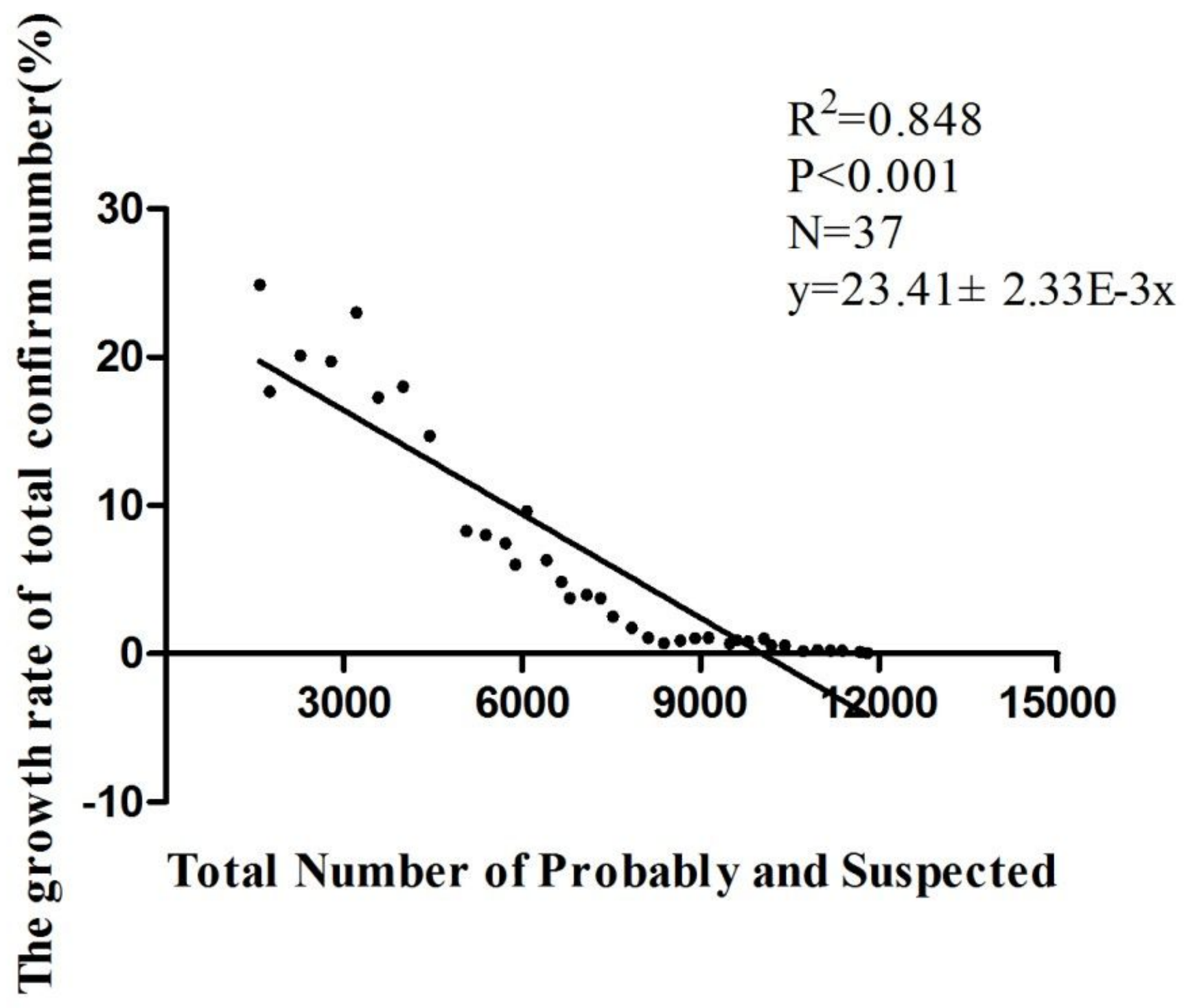

Figure 5

The correlation analysis between quarantine before infection and the weekly EVD increase rates in Liberia 


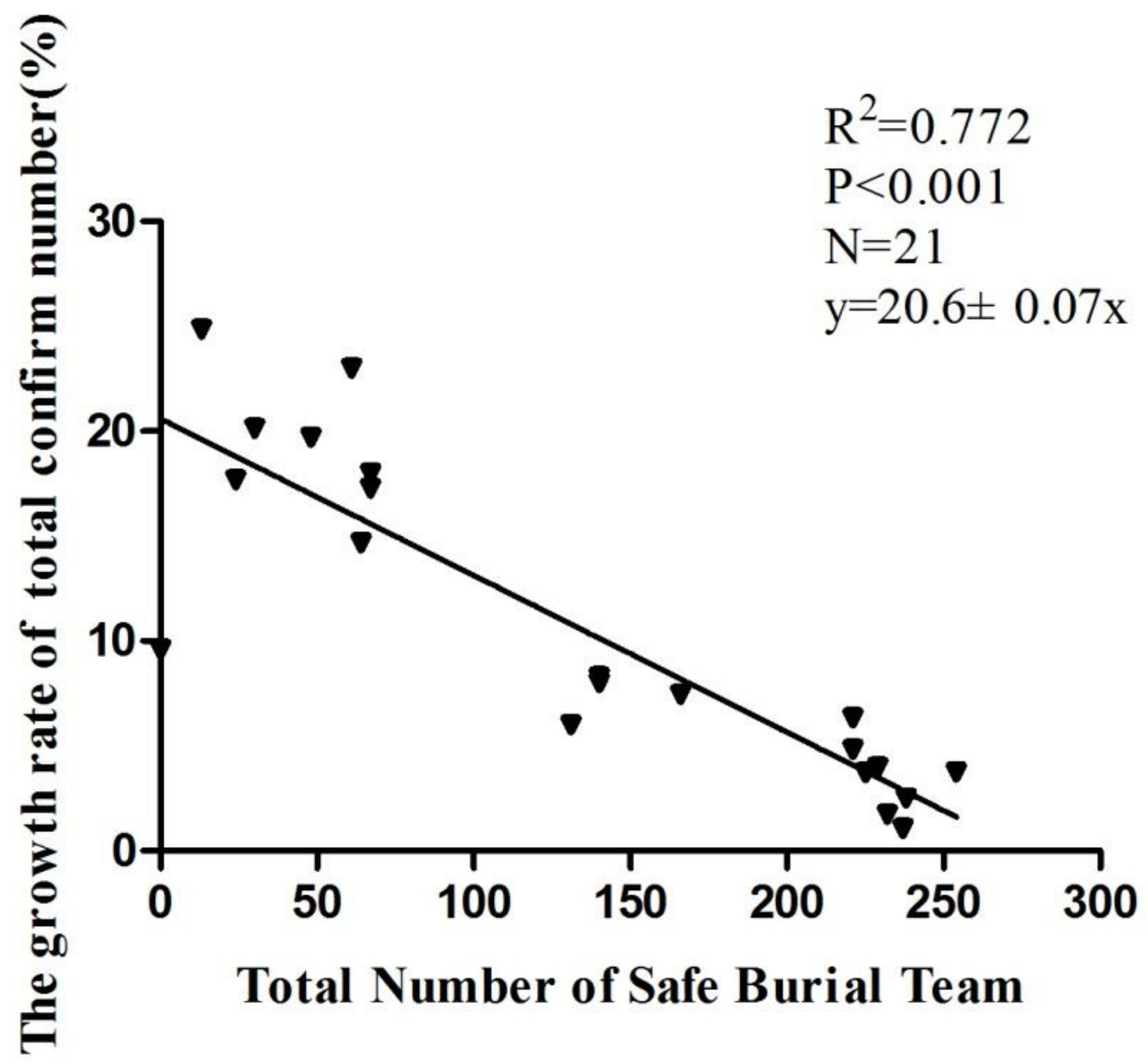

Figure 6

The correlation analysis between safe burial teams and the weekly EVD increase rates in Sierra Leone 


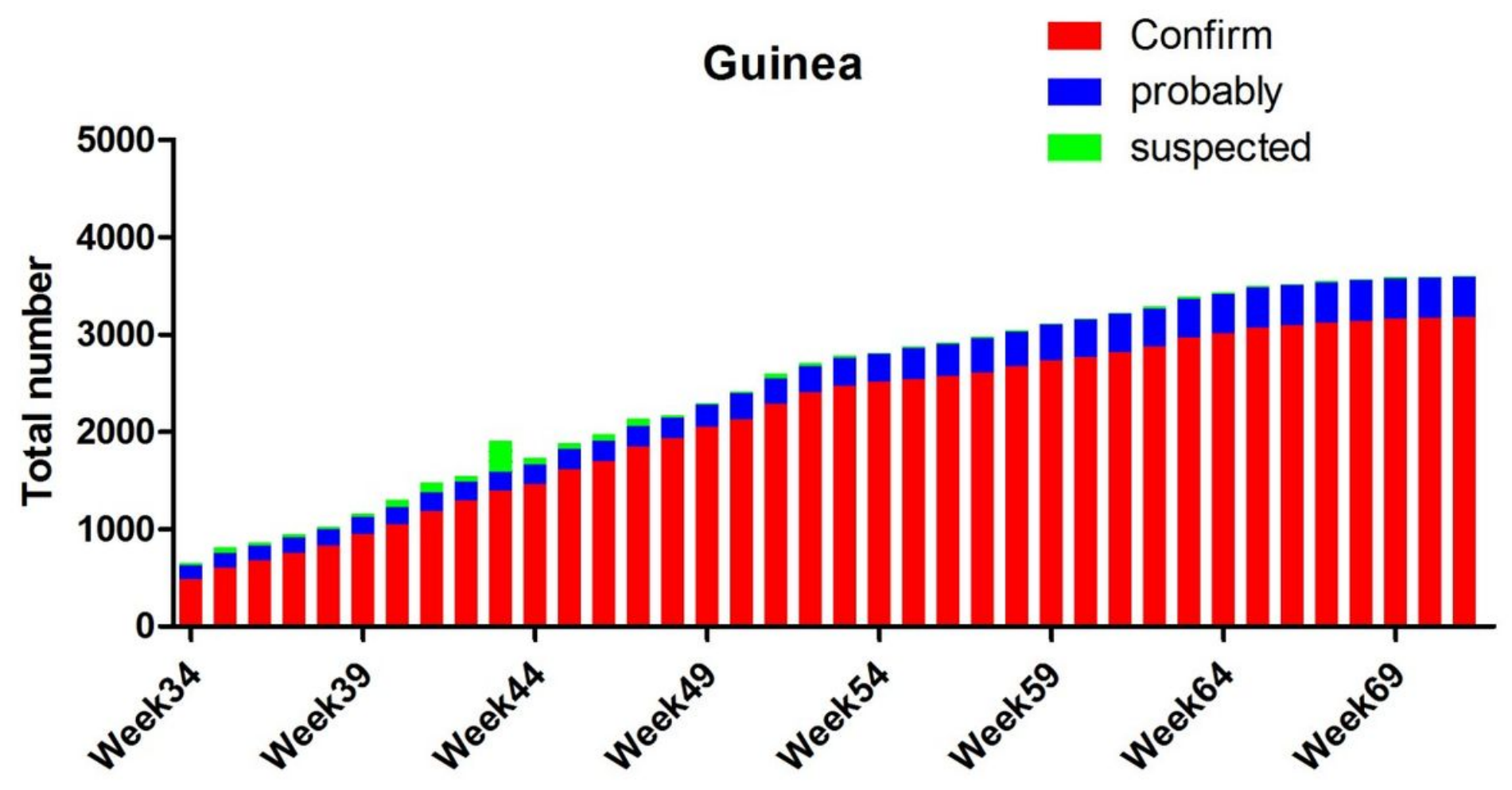

Figure 7

The quarantine number before EVD outbreak in Guinea

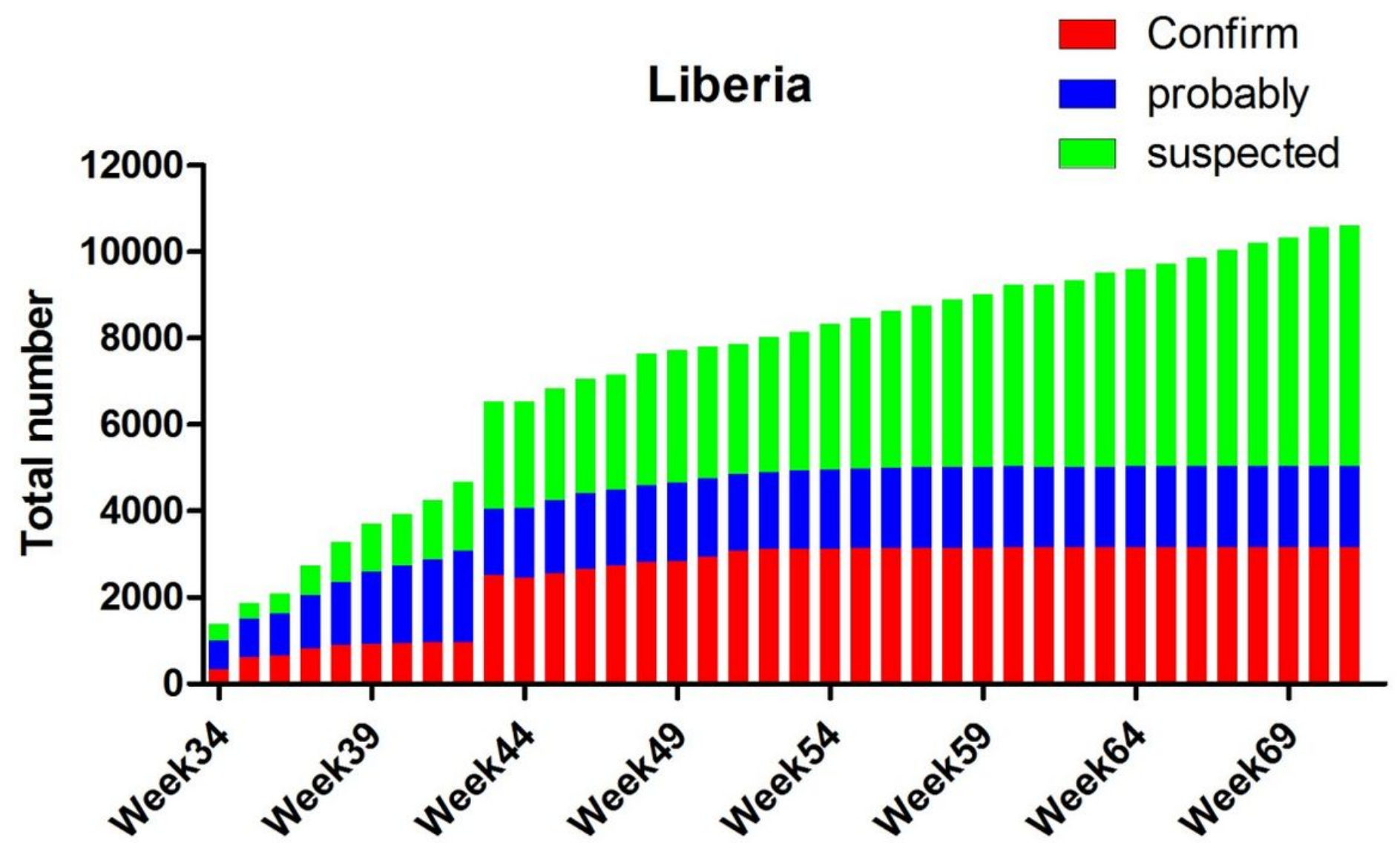


Figure 8

The quarantine number before EVD outbreak in Liberia
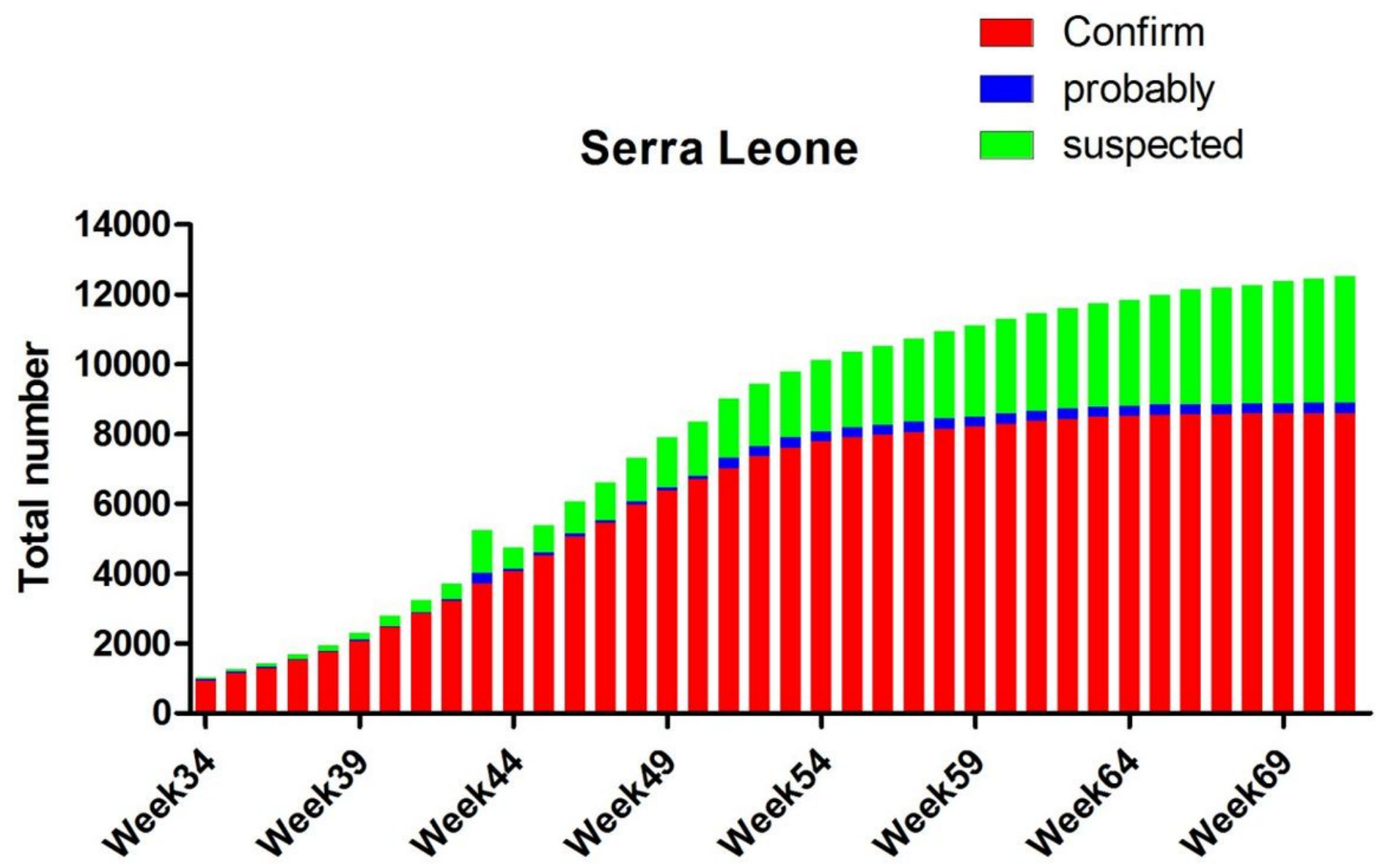

Figure 9

The quarantine number before EVD outbreak in Sierra Leone

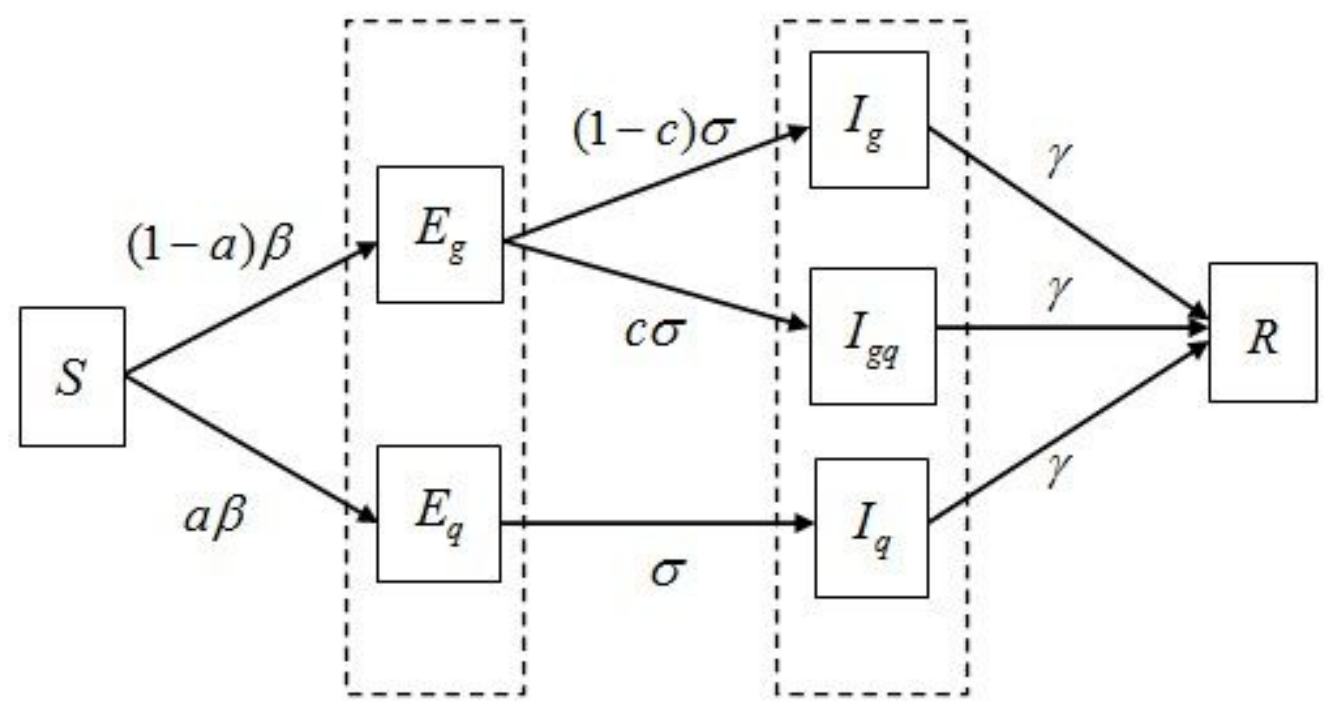


Figure 10

State transitions of individuals in the SEfEclfIclfcR model 